\title{
Diseño de un curso virtual de alfabetización digital para docentes de la Universidad de la Amazonia
}

\section{Design of a virtual digital literacy course for teachers of the University of the Amazonia \\ Projeto de um curso virtual de alfabetização digital para professores da Universidade da Amazônia}

\author{
Cindy Tatiana Guayara Cuéllar ${ }^{1}$ \\ Edwin Eduardo Millán Rojas ${ }^{2}$ \\ Carlos Alberto Gómez Cano ${ }^{3}$
}

Recibido: mayo de 2018

Aceptado: noviembre de 2018

Para citar este artículo: Guayara-Cuéllar, C. T., Millán-Rojas, E. E. y Gómez-Cano, C. A. (2019). Diseño de un curso virtual de alfabetización digital para docentes de la Universidad de la Amazonia. Revista Científica, 34(1), 34-48. Doi: https://doi.org/10.14483/23448350.13314

\section{Resumen}

El presente artículo da cuenta de una investigación desarrollada cuyo objetivo general fue desarrollar un curso virtual de alfabetización digital para mejorar las competencias en el uso de las tecnologías de la información y las comunicaciones (TIC) de los docentes de la Universidad de la Amazonia de Florencia (Caquetá, Colombia). Esto con la intención de proponer una estrategia para reforzar la alfabetización digital. El desarrollo metodológico general se hizo desde los métodos exploratorio y proyectivo, utilizando una muestra de 100 docentes. Para establecer la metodología específica se definió: la primera fase (diagnóstico del problema), la segunda fase (el contenido teórico) y la tercera fase (diseño e implementación del curso virtual). Con los resultados obtenidos, la Universidad de la Amazonia contará con una estrategia para tratar el problema de alfabetización digital y competencias TIC en los docentes y a nivel nacional podrá ser un referente para implementar y mejorar las competencias TIC en los docentes universitarios.

Palabras clave: competencias TIC, educación, informática educativa, UDLA, didáctica.

\begin{abstract}
Nitrogen compounds are being injected into the environment by human activities, which are altering the global nitrogen cycle and, thus, water systems. There is principally serious growing concern about the actual environmental consequences of the nitrogen pollutants in groundwater since a safe supply of clean water is needed for human consumption. Recent observations in the field of measurements and studies in the laboratory suggest that there are limitations of effective methods that detect and quantify
\end{abstract}


biogeochemical reactions of nitrogen loss occurred in this aquatic body. This review explores our understanding of nitrogen pollutants on groundwater, taking into consideration the biogeochemical reactions of nitrogen cycle, and the limitations and advantages of analytical methods used in the detection of nitrogen compounds.

Keywords: education, educational computing, TIC skills, UDLA, didactic.

\section{Resumo}

Este artigo tem como objetivo geral desenvolver um curso de literacia digital virtual para melhorar as habilidades no uso da tecnologia da informação e comunicação (TIC) no ensino da Universidade da Amazônia Florença Caquetá. O acima exposto com o propósito de propor uma estratégia para reforçar a alfabetização digital. O desenvolvimento metodológico geral foi abordado a partir dos métodos exploratório e projetivo, utilizando uma amostra de 100 professores. Para definir a metodologia específica definida: a primeira fase, o diagnóstico de problemas, a segunda fase, o conteúdo teórico ea terceira fase, design e implementação do curso virtual. Universidade da Amazônia pode ter uma estratégia para abordar as competências de literacia digital e TIC entre professores e nacional pode ser uma referência para implementar e melhorar competências em TIC no ensino universitário.

Palavras-chaves: competências TIC, educação, informática educativa, UDLA, didáctica.

\section{Introducción}

Las tecnologías de la información y la comunicación (TIC) se han incorporado en todas las actividades que realiza el ser humano en sus quehaceres cotidianos revolucionando y transformando al mundo todos los días. Unas de las contribuciones más importantes que han hecho las TIC ha sido al mejoramiento del sistema educativo. No obstante, más allá del impacto que genera dicha evolución tecnológica, está también trajo consigo aspectos a superar, principalmente en los docentes, pues una vez implementadas en la educación se requiere la capacitación de los mismos y el fortalecimiento en competencias digitales, herramientas web 2.0 y uso y manejo de las TIC. Como medio, las TIC apoyan favorablemente cualquier disciplina, pero la utilización inadecuada e incorrecta puede generar barreras que impiden el normal desarrollo de lo mencionado con anterioridad. La educación superior es un bastión para desarrollar tecnologías emergentes que medien en la educación, pero no es ajena a los problemas mencionados. La Universidad de la Amazonia en sus casi 40 años de existencia se ve enfrentada al cambio generacional de sus docentes. Los cambios no siempre son bien recibidos y es necesario realizar procesos para mejorar la educación. Por ello, se tuvo como objetivo general desarrollar un curso virtual de alfabetización digital para docentes de la Universidad de la Amazonia.

El problema del bajo nivel de alfabetización digital de los docentes universitarios se centra en la no inclusión de las TIC en las labores académicas. Según Olivé (2005), esta nueva connotación, augura en la educación que en un futuro se pasará de una sociedad informada a una sociedad más formada, tanto en el ámbito cultural como en el educativo; por lo que el adecuado uso de las TIC será una condición sustancial para el desarrollo de la sociedad del conocimiento. También se considera que las TIC sirven a la docencia al utilizar herramientas pedagógicas, equipos y medios de comunicación en los programas y soluciones para las personas (Tilve, 2007).

El estado actual de alfabetización digital en los docentes universitarios se evaluó bajo las competencias descritas por el Ministerio Nacional de Educación, las cuales tienen como propósito la guía en el proceso de desarrollo profesional docente para la innovación educativa pertinente utilizando las TIC. Para conocer su nivel y garantizar la calidad de la educación superior, los lineamientos están dirigidos tanto para quienes diseñan e implementan los programas de formación, como para los docentes y directivos docentes en ejercicio (MEN, 2017). 
El método utilizado para el desarrollo de la investigación es tanto exploratorio como proyectivo; este último busca la elaboración de la propuesta del curso virtual de alfabetización digital para docentes de la Universidad de la Amazonia. Como resultado, se presenta la producción del curso virtual desarrollado por etapas, tal como lo establece el Ministerio de Educación Nacional (2013) en la publicación "Orientaciones para el diseño, producción e implementación de cursos virtuales", en el que se define que el curso virtual debe facilitar la construcción de procesos formativos que permitan el desarrollo de las actividades de aprendizaje propuestas al estudiante, así como el seguimiento y evaluación que realice el docente (Calderón, Buitrago, Acevedo y Tobón, 2013).

\section{Metodología}

La metodología propuesta para desarrollar el curso virtual de alfabetización digital para los docentes en la Universidad de la Amazonia se basa en una perspectiva que comprende dos tipos de investigación. El primero, es una investigación exploratoria la cual se utilizó para indagar sobre el estado actual de la alfabetización digital de docentes universitarios; asimismo, para establecer los referentes teóricos que sustenten el desarrollo del curso virtual y la búsqueda de los contenidos teóricos. El segundo, el método proyectivo utilizado en la elaboración de la propuesta del curso virtual de alfabetización digital. El enfoque utilizado en el desarrollo de la metodología fue el mixto cualitativo-cuantitativo que, según Binda y Balbastre-Benavent (2013), recolecta, analiza y vincula datos cuantitativos y cualitativos en un mismo estudio.

La investigación se desarrolló en tres fases. La primera comprende el diagnóstico del problema, en la cual se obtuvo material bibliográfico sobre las competencias en el uso de las TIC y la alfabetización digital para los docentes universitarios. En la segunda fase se elaboró el contenido teórico, se realizaron talleres, actividades y evaluaciones para complementar la estrategia pedagógica. Por último, en la tercera fase se diseñó e implementó del curso virtual, elaborando las actividades de desarrollo para fortalecer el conocimiento y habilidades en los docentes universitarios.

Durante la etapa del diagnostico se aplicó una encuesta en la recolección de información para determinar el nivel de alfabetización digital de los docentes de la Universidad de la Amazonia. La encuesta incluyo cinco preguntas con única respuesta catalogadas de la siguiente forma: para la respuesta de las preguntas uno y cinco, se definió una escala numérica de uno a cinco, donde cinco es el valor mayor; para la respuesta dos, se definió una escala descriptiva de mucho, poco o nada; para la respuesta de la pregunta tres se definió una escala binaria de si o no y para la respuesta a la cuarta pregunta se definió la escala descriptiva con siete opciones las cuales fueron: ExeLearning, Prezi, CmapTools, Edmodo, Socrative, Ninguna u Otros. La muestra de estudio está conformada por 100 profesores en diferentes áreas del conocimiento de la Universidad de la Amazonia (de un universo de 712 profesores), donde 77 de los encuestados son hombres y 23 mujeres entre los 25 y 55 años de edad.

Para el desarrollo el curso virtual se utilizó la plataforma Moodle del Campus Virtual presencial (Aula extendida) de la Universidad de la Amazonia, integrando herramientas de desarrollo como: Educaplay para las actividades de aprendizaje y Adobe Captative, bajo la licencia de uso académico para reforzar conceptos y definiciones.

\section{Resultados}

Para el análisis de los datos obtenidos, y con el fin de tener mayor claridad en la información recolectada, se presenta la figura 1.

De acuerdo a la figura 1, solamente el $39 \%$ de los encuestados se consideran profesores no alfabetizado digitalmente. Sin embargo, en las categorías regular y aceptable se presentan un $27 \%$ de la muestra, no obstante combinada con la categoría 
No bueno representan el $63 \%$ de los profesores encuestados, los cuales consideran no tener las competencias adecuadas para el uso de las TIC en el aula de clase. El $24 \%$ de los entrevistados considera tener muy buenas competencias en el manejo de las TIC y el $9 \%$ cree tener una capacidad media alta en el manejo y uso de las tecnologías en el salón de clase.

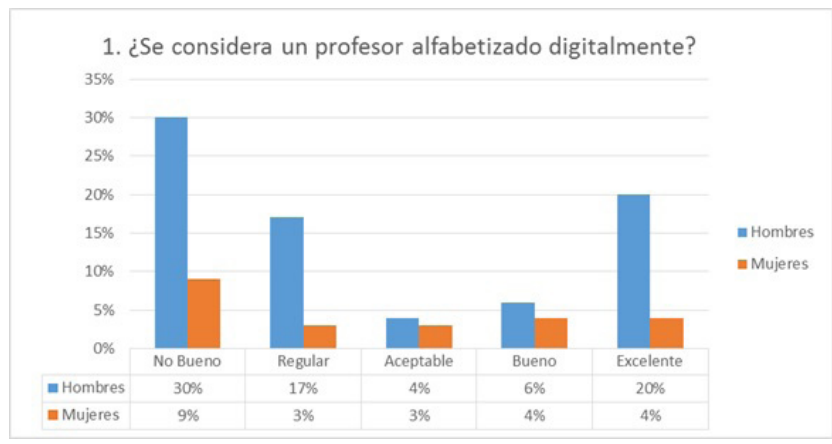

Figure 1. ¿Se considera un profesor alfabetizado digitalmente?, porcentajes del grado de alfabetización.

Fuente: elaboración propia.

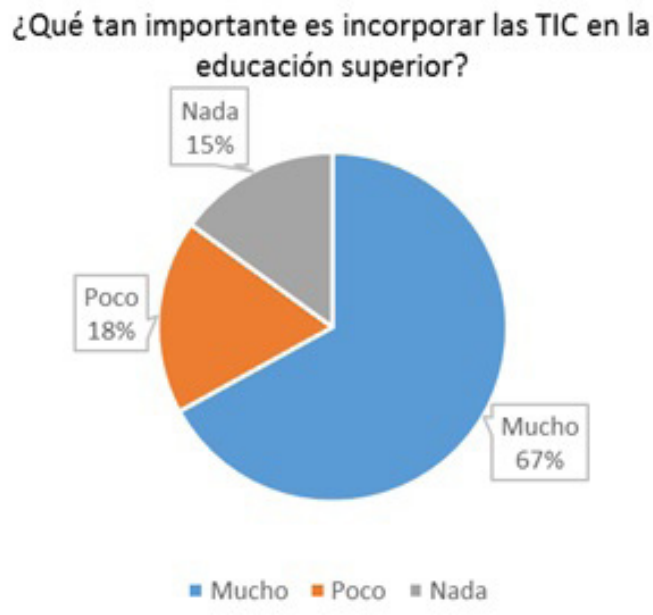

Figura 2. Grado de importancia de las TIC en la educación superior.

Fuente: elaboración propia.

Se evidencia que el $67 \%$ de los encuestados (que equivale a 67 docentes) considera que es importante incorporar las TIC en la educación superior. El $15 \%$, por el contrario, cree en la no utilización de las TIC en los procesos de enseñanza y aprendizaje; y el $18 \%$ le da poca importancia a las tecnologías.

\section{¿Implementa alguna herramienta tecnológica a la hora de orientar sus clases?}

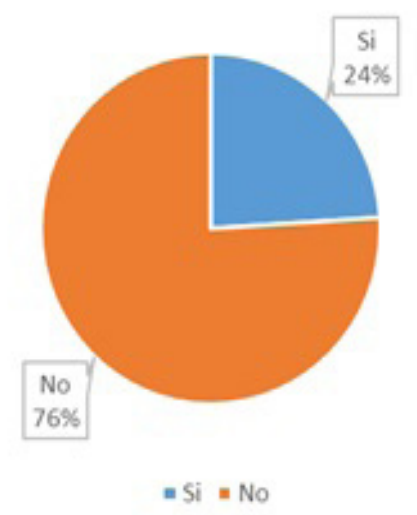

Figura 3. Porcentaje de uso de las TIC en el aula.

Fuente: elaboración propia.

Con base en los resultados obtenidos en la pregunta número tres, se observa que el $76 \%$ no implementan por lo menos una herramienta tecnológica en las clases; mientras el $24 \%$ de los docentes sí logran hacerlo y las Ilevan a la práctica en el aula de clase.

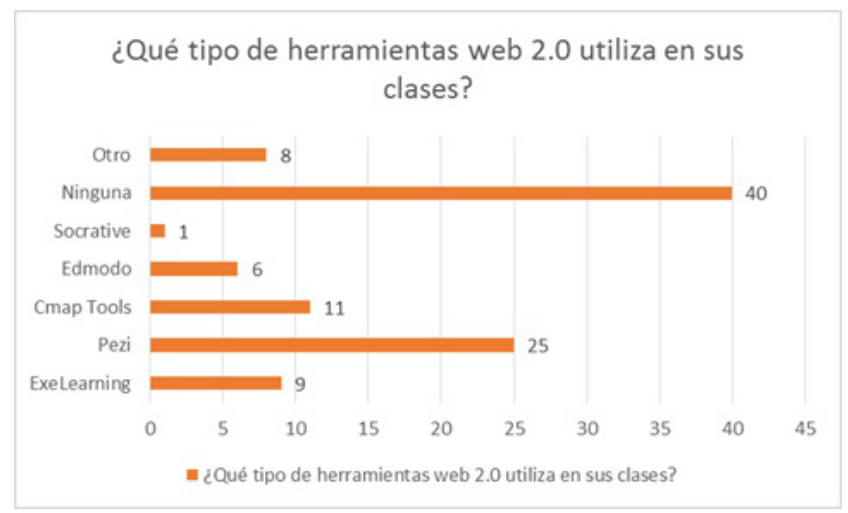

Figura 4. Uso de las herramientas web 2.0.

Fuente: elaboración propia de los autores.

Según las estadísticas, un $40 \%$ de los docentes no utiliza ningún tipo de herramienta web 2.0 en sus clases. La herramienta líder en los resultados (con un $25 \%$ ) es usada para presentaciones; enseguida (con $11 \%$ ) la herramientas de mapas 
mentales. Las demás presentan un bajo uso por parte de los docentes.

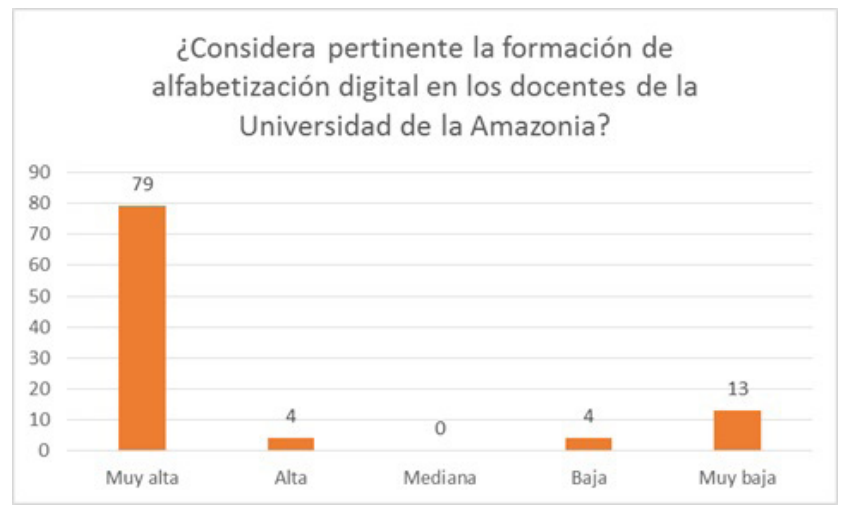

Figura 5. Grado de pertinencia de la alfabetización digital.

Fuente: elaboración propia.

De acuerdo con las preguntas analizadas en la figura 5, el 79\% de los encuestados contestaron, sí es pertinente la alfabetización digital. Sin embargo, persiste un 13\% de docentes con muy baja pertinencia en la formación docente en competencias TIC; y solo un 4\%, la considera baja. Una vez finalizada la aplicación de la encuesta a los docentes, se procedió a la exploración de los referentes teóricos para proceder a las siguientes fases: diseño, planeación e implementación del curso virtual de alfabetización digital en la plataforma Moodle en la Universidad de la Amazonia.

\section{Referentes teóricos para el desarrollo de un curso virtual de alfabetización digital de los docentes}

Se exploraron diferentes fuentes bibliográficas y se consolidó una base para el desarrollo del curso virtual de alfabetización digital de los docentes. Es de aclarar la necesidad de mantener un trabajo constante para brindar una actualización y mejora al proceso de aprendizaje ofrecido. Los elementos de la tabla 1 reflejan el trabajo inicial para la elaboración del diseño y la planificación del espacio académico (curso).

Tabla 1. Referentes teóricos para el diseño del curso virtual de alfabetización.

\begin{tabular}{|c|c|}
\hline Material bibliográfico & Descripción \\
\hline $\begin{array}{l}\text { Lineamientos para la formulación de planes } \\
\text { estratégicos de incorporación de tecnologías } \\
\text { de la información y comunicación (TIC) en } \\
\text { instituciones de educación superior (IES). }\end{array}$ & $\begin{array}{l}\text { "Busca responder al interés del Ministerio de acompañar a las } \\
\text { IES en la formulación de estrategias de uso de MTIC en los } \\
\text { Planes Institucionales". (Osorio, y otros, 2008, págs. 13-15) }\end{array}$ \\
\hline $\begin{array}{l}\text { Orientaciones para el diseño, producción e } \\
\text { implementación de Cursos Virtuales }\end{array}$ & $\begin{array}{l}\text { "Propósitos adelantar la sistematización y documentación de } \\
\text { sus proyectos, iniciativas y experiencias". (MEN, 2017) }\end{array}$ \\
\hline $\begin{array}{l}\text { El profesorado universitario en la sociedad } \\
\text { del conocimiento: competencias profesionales } \\
\text { docentes }\end{array}$ & $\begin{array}{l}\text { "Proporcionar una recopilación teórica y reflexiva acerca de } \\
\text { las competencias profesionales que el docente universitario } \\
\text { debe tener para satisfacer las necesidades que la sociedad del } \\
\text { conocimiento demanda de la universidad del siglo XXI". (Bozu } \\
\text { \& Canto, 2009) }\end{array}$ \\
\hline $\begin{array}{l}\text { Competencias TIC para el desarrollo profesional } \\
\text { docente. }\end{array}$ & $\begin{array}{l}\text { "Orientar los procesos de formación en el uso de TIC que se } \\
\text { estaban ofreciendo a los docentes del país". (MEN, 2017) }\end{array}$ \\
\hline $\begin{array}{l}\text { Curso certificación maestros públicos. Desarrollo } \\
\text { de unidades. }\end{array}$ & $\begin{array}{l}\text { "Iniciativa del Ministerio de Tecnologías de la Información y la } \\
\text { Comunicación, para promover la ciudadanía digital mediante } \\
\text { el acceso, uso y apropiación masiva de las TIC, entre los } \\
\text { maestros y servidores públicos e incrementar los niveles de } \\
\text { incorporación, adaptación e integración de estas tecnologías } \\
\text { en los servicios del Gobierno y sector educativo". (UNAD- } \\
\text { MINTIC, 2018) }\end{array}$ \\
\hline
\end{tabular}

Fuente: elaboración propia de los autores. 


\section{Fase de diseño del curso virtual}

El diseño, producción e implementación de un curso virtual es una tarea que requiere la elaboración de procedimientos, estructuras, herramientas, recursos y aplicación de estándares para la generación y organización de escenarios virtuales que promuevan aprendizaje de calidad, es decir, que garanticen el cumplimiento de los propósitos de formación definidos para el curso (García, 2016). Lo anterior, con el fin de facilitar la construcción de procesos de formación que permitan el desarrollo de actividades de aprendizaje propuestas al estudiante, así como el seguimiento y evaluación que realiza el docente (Vergel-Ortega, Martínez-Lozano y Zafra-Tristancho, 2016).

El diseño, producción e implementación de un curso virtual puede estar trazado por fases o etapas que atienden al análisis de necesidades y expectativas de formación o responden a los objetivos y propósitos de enseñanza y de aprendizaje. Estos, definidos desde su propuesta curricular (MEN, 2017).

Las etapas para el diseño, producción e implementación se pueden ver en la figura 6 .

\section{Diagnóstico y planeación}

Actualmente se requieren de nuevos conceptos para definir la alfabetización digital. En la literatura especializada existe un gran debate en torno a la conceptualización del término, pues este solo se limita a todo lo relacionado con el uso de la información, el internet, las redes y el computador. El adjetivo digital se refiere no solo a las habilidades para el adecuado uso de internet, sino también para usar documentos hipertextuales.
En este sentido, (Gilster \& Glister, 1997, pág. 139) sostienen que: "la alfabetización digital es un conjunto de conocimientos, habilidades y competencias que es necesario adquirir para el uso funcional y constructivo de las TIC, por lo tanto, se concluye que la alfabetización digital es la construcción de conocimiento a través de distintas fuentes y herramientas y no solo orientado a la web". La Unesco (2005) enmarca esta necesidad en el ámbito de la educación superior, señalando la necesidad de la competencia digital docente en el conocimiento básico de la tecnología digital, herramientas de comunicación, uso de una amplia gama de textos para expresar ideas propias a través de medios diversos, así como la búsqueda de información y entendimiento de los propósitos de los jóvenes y el uso de internet.

\section{Tipos de recursos y actividades}

El curso virtual está compuesto por cuatro módulos. El primer módulo tiene como tema el delito informático y los subtemas de hackeo, suplantación de identidad y phishning. El segundo módulo es sobre riesgos en internet con los siguientes subtemas: ciberacoso, sexting y grooming. El tercer módulo es de herramientas web 2.0, con los subtemas de OVAS y REAS; cada uno cuenta con material de conceptualización, características y videos. Finalmente, el módulo cuatro es de evaluación y en este se encontrará la retroalimentación de cada uno de los módulos anteriores.

En la introducción se encuentra una breve presentación de los elementos y referentes teóricos utilizados en el curso de alfabetización digital. En la sección de objetivos están manifiestos los propósitos a lograr por parte de los docentes universitarios en el desarrollo del curso.

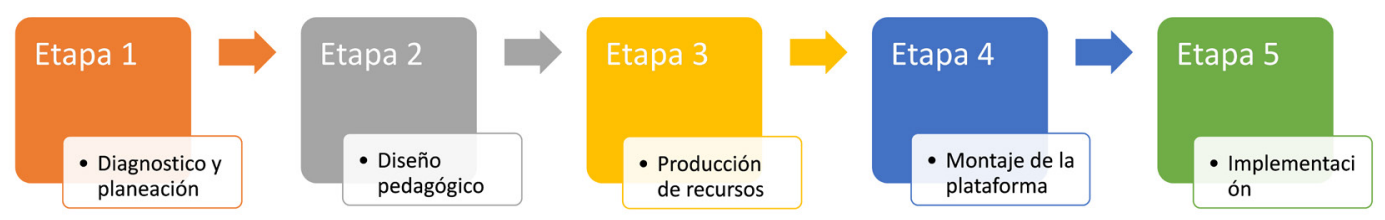

Figura 6. Método de elaboración de cursos virtuales.

Fuente: MEN (2017). 


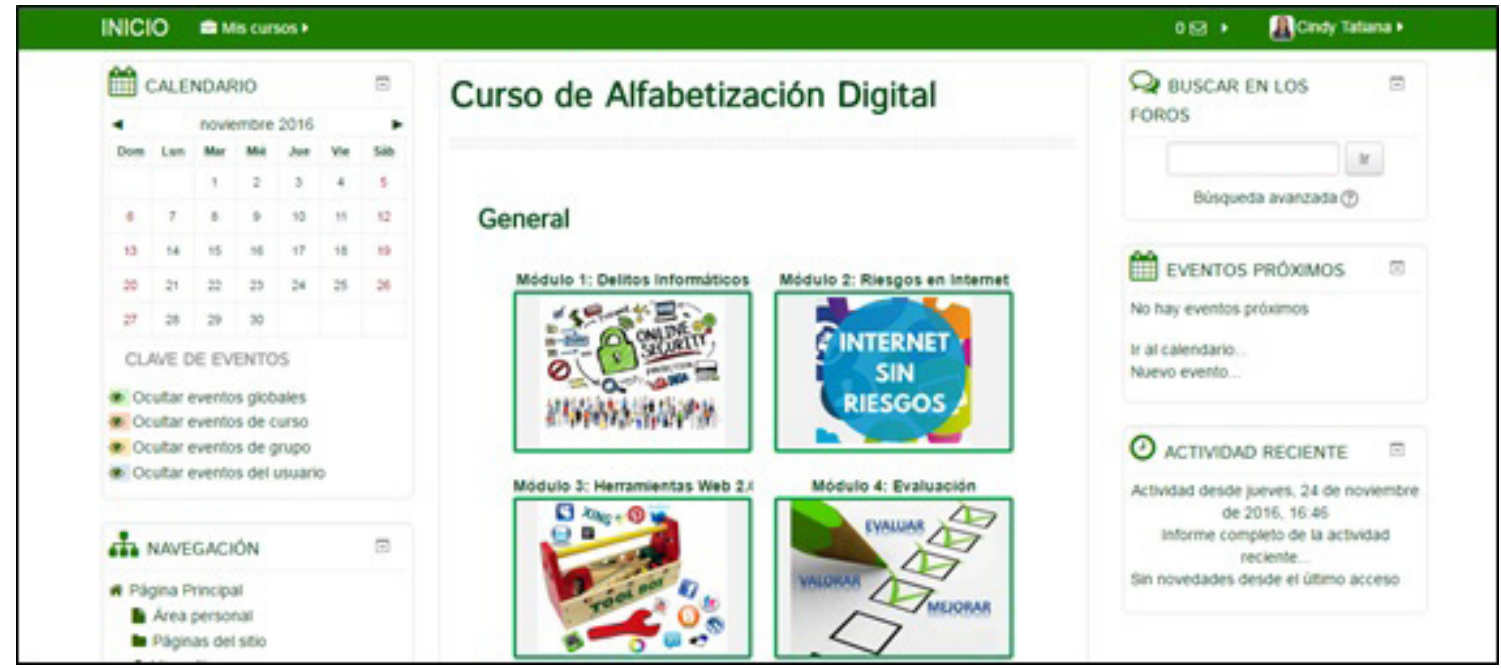

Figura 7. Módulos del curso virtual.

Fuente: Plataforma aula extendida Uniamazonia, Moodle.

\section{Diseño pedagógico}

Un ambiente de enseñanza y de aprendizaje es un escenario físico o virtual diseñado por el docente, cuya intención es el logro de unos objetivos de enseñanza y aprendizaje concretos a través de la articulación de diversas estrategias, métodos, actividades y recursos educativos. Esto significa un proceso reflexivo donde se atienden las preguntas del qué, cómo y para qué se enseña (Coll, 2014). Los aspectos a tener en cuenta para el desarrollo del curso virtual con el modelo pedagógico institucional (Uniamazonia, 2017) comprende los siguientes elementos: autoaprendizaje, material didáctico, espacio de comunicación y encuentros presenciales bajo la plataforma Moodle. Para lograr lo mencionado, fue necesario contar con un equipo de trabajo interdisciplinar en el diseño y producción de ambientes virtuales para lograr el desarrollo de un plan de formación docente en competencias TIC y digitales haciendo uso de los recursos educativos de la IES.

\section{Producción de recursos y modelado educativo}

Con la llegada de las nuevas tecnologías, es cada vez más fuerte la tendencia en donde el profesional docente cambia el enfoque clásico de dictar las clases al lado del tablero y haciendo uso del discurso basado en clases magistrales a un enfoque de formación centrado en un entorno interactivo de aprendizaje. En este sentido, la Unesco (2005) sustentó en el área educativa los objetivos para mejorar la calidad de la educación por medio de la innovación, la difusión y el uso compartido de información y de buenas prácticas, utilizando múltiples contenidos y métodos.

Se utilizaron recursos educativos abiertos como videos, juegos, actividades, entre otros, que permitieron enriquecer cada uno de los módulos diseñados. Sin embargo, la Universidad de la Amazonia cuenta con la infraestructura tecnológica para la creación de nuevo material audiovisual en las áreas de enseñanza de los docentes.

El diseño de los elementos del curso se realizó por módulos. El contenido de cada módulo se puede ver en la figura 8.

En esta primera fase se cuenta con los recursos mencionados previamente. Pero, es necesario dejar claro que el curso puede ser alimentado según las necesidades del docente orientador o del material generado por los mismos estudiantes. 

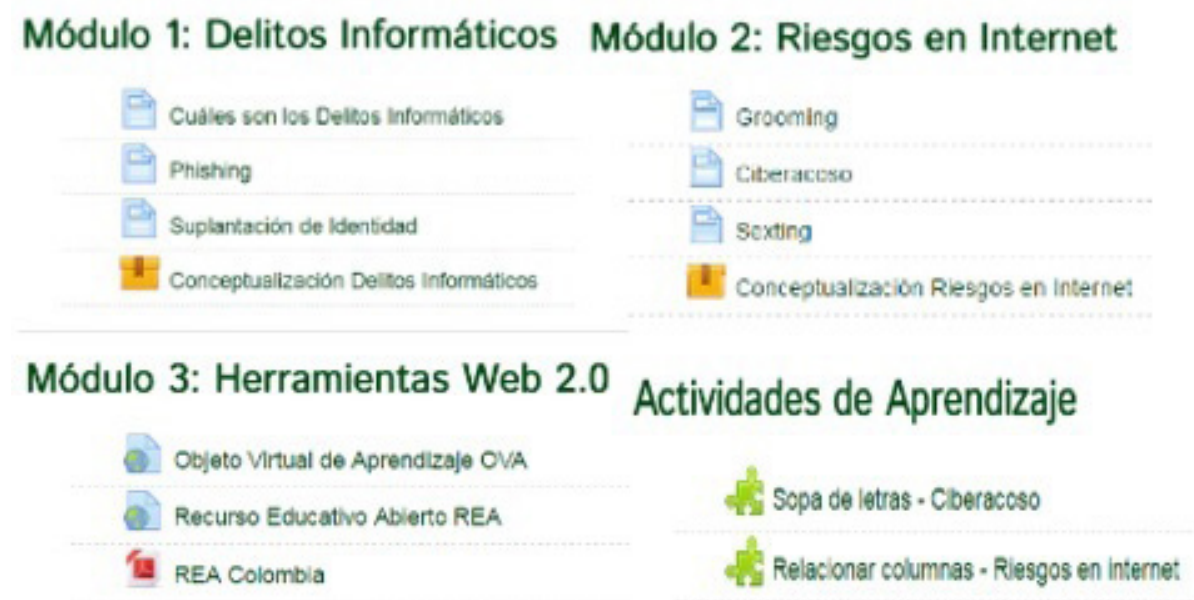

\section{Evaluación}

Figura 8. Actividades desarrolladas por módulos.

Fuente: elaboración propia.

\section{Plataforma}

La plataforma seleccionada fue moodle, pues varios autores ${ }^{4}$ la consideran como una herramienta versátil y de una utilidad sin precedentes en las plataformas educativas. A partir de la definición de 15 símbolos plenamente significativos se puede desarrollar todo el proceso de enseñanza de diversos temas.

La plataforma moodle permite la retroalimentación y el seguimiento del proceso de enseñanza con los estudiantes, los cuales contribuyen de forma colaborativa a su enseñanza. Por otra parte, el sistema de evaluación es propuesto por el docente tutor de acuerdo al modelo utilizado y a las necesidades determinadas en los estudiantes.

Entre ellos: Iglesias-Rodríguez, Olmos-Migueláñez, Torrecilla-Sánchez y Mena-Marcos (2014), Rodríguez, Migueláñez, Sánchez y Marco (2015), Salas, Barrera y Fuentes (2015) y López (2015)

\section{Implementación del curso virtual}

El desarrollo del curso se llevó a cabo por módulos. A continuación, se presenta un diseño preliminar de los elementos utilizados por los participantes del curso virtual.

En el módulo 1 se encuentran cuatro actividades relacionadas con el tema de delitos informáticos. A continuación, se presenta la descripción de cada una.

En este primer tema encontramos un video del MinTIC presentado por el programa de "En TIC confío" en el que se definen los tres delitos informáticos básicos: hackeo, suplantación de identidad y phishing.

Para el segundo tema, sobre phishing, se usó un video elaborado por C\&M consultores en el cual se explica la forma en la que se puede ser víctima y una breve descripción del mismo.

Además de lo ya expuesto, se tiene elaborado en la herramienta Adobe Captative una presentación 
de conceptualización sobre los delitos informáticos muy intuitiva y fácil de manejar. En esta se encuentra una evaluación para ser realizada en un documento de Word y posteriormente subir los resultados en la sección de Evaluación. En el módulo 2 se encuentran cuatro actividades relacionadas con el tema de riesgos en internet. A continuación, se hace la descripción de cada una de ellas.

El primer tema sobre grooming se explica haciendo uso de un video elaborado por Colombia Digital. Allí expresan la forma para ser víctima de grooming y presentan una breve descripción del mismo.

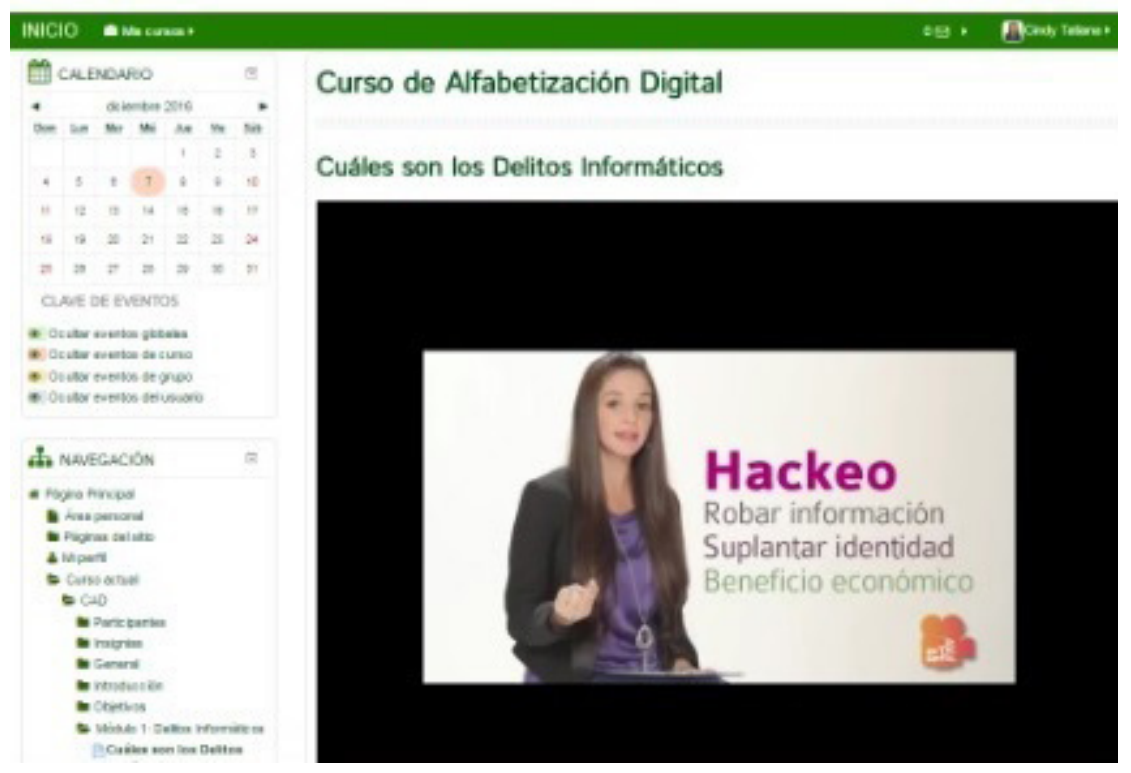

Figura 9. Delitos informáticos.

Fuente: (MinTIC, 2017).

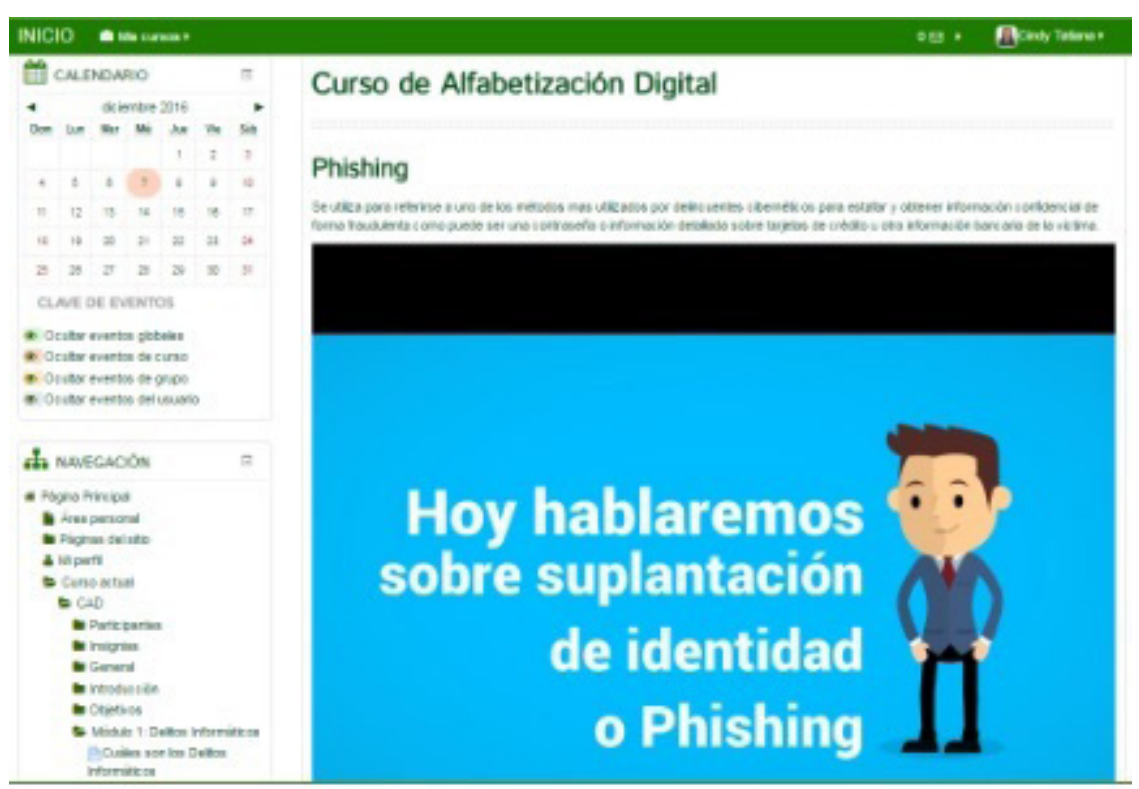

Figura 10. Video en el que se define phishing.

Fuente: (C\&M consultores, 2017). 


\section{Conceptualización Delitos Informáticos}

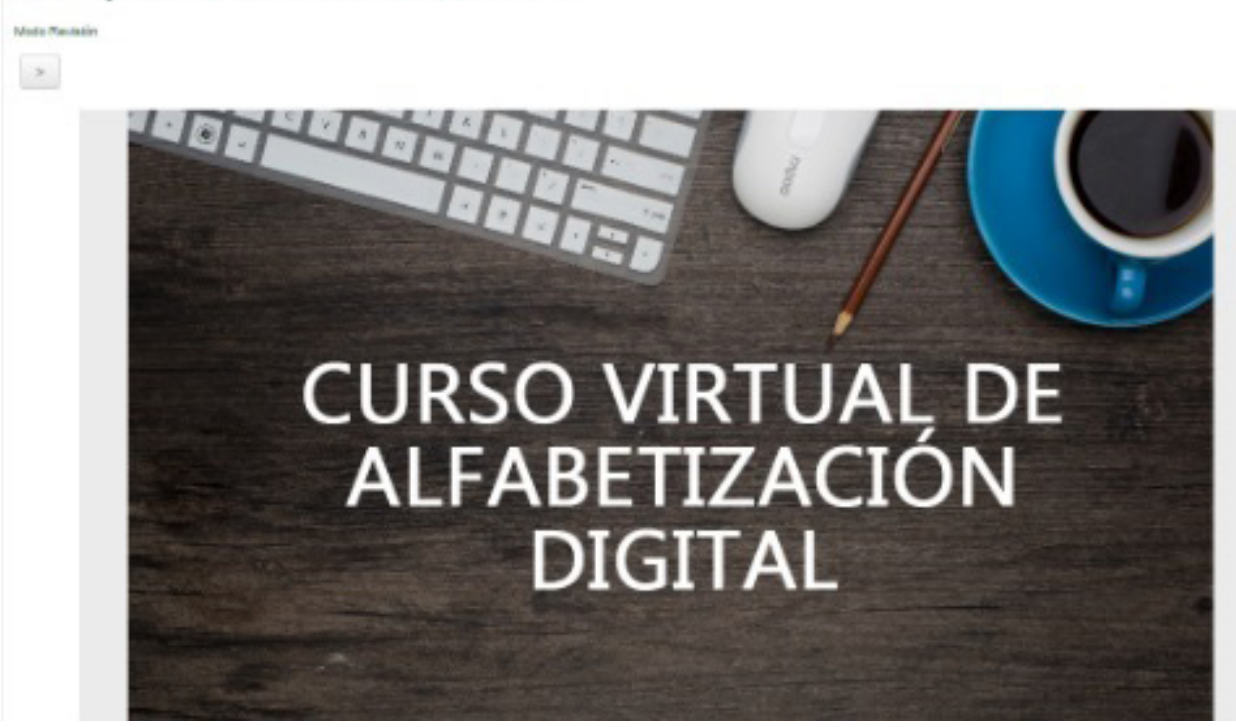

Figura 12. Presentación de conceptualización sobre los delitos informáticos.

Fuente: elaboración propia.

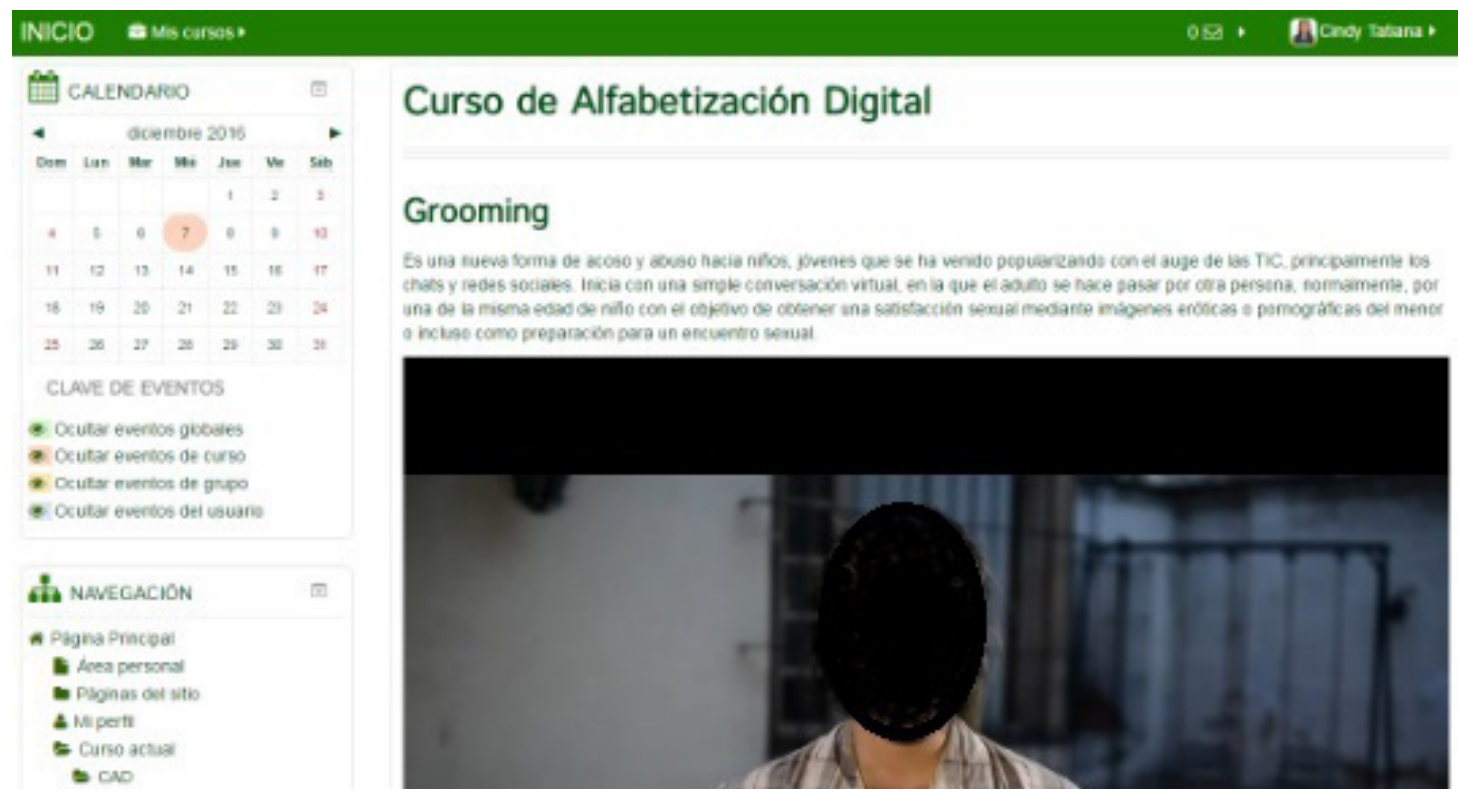

Figura 13. Definición de grooming.

Fuente: (Colombia digital, 2017).

Para el tema de ciberacoso se usó un video que circulo en En TIC confío en que se explica la forma para ser víctima de ciberacoso y una breve descripción del mismo
El tercer tema abordado es sexting. Este se abordó haciendo uso de un video elaborado por Pantallas Amigas, donde explican la forma para ser víctima de sexting y una breve descripción del mismo. 


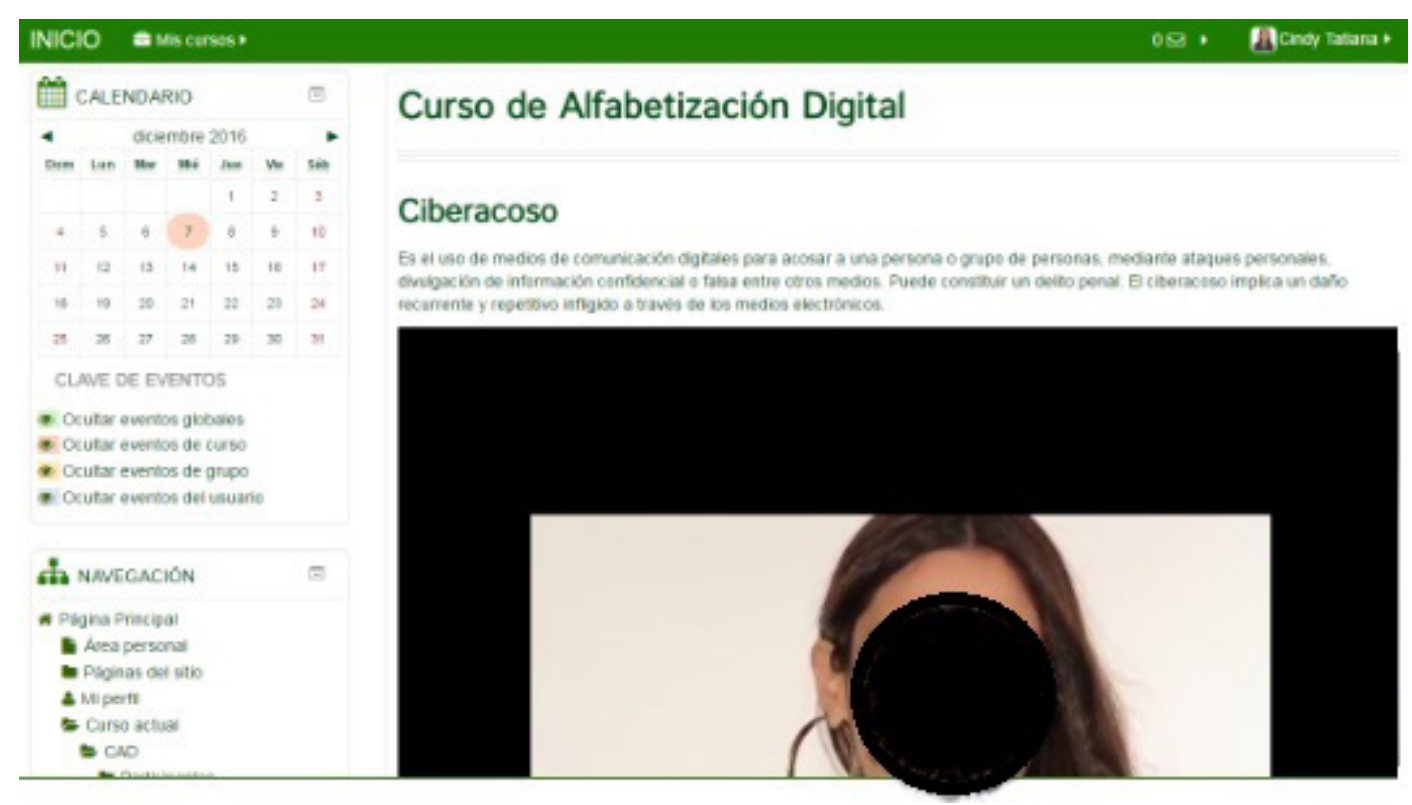

Figura 14. Definición de ciberacoso.

Fuente: (MinTIC, 2017).

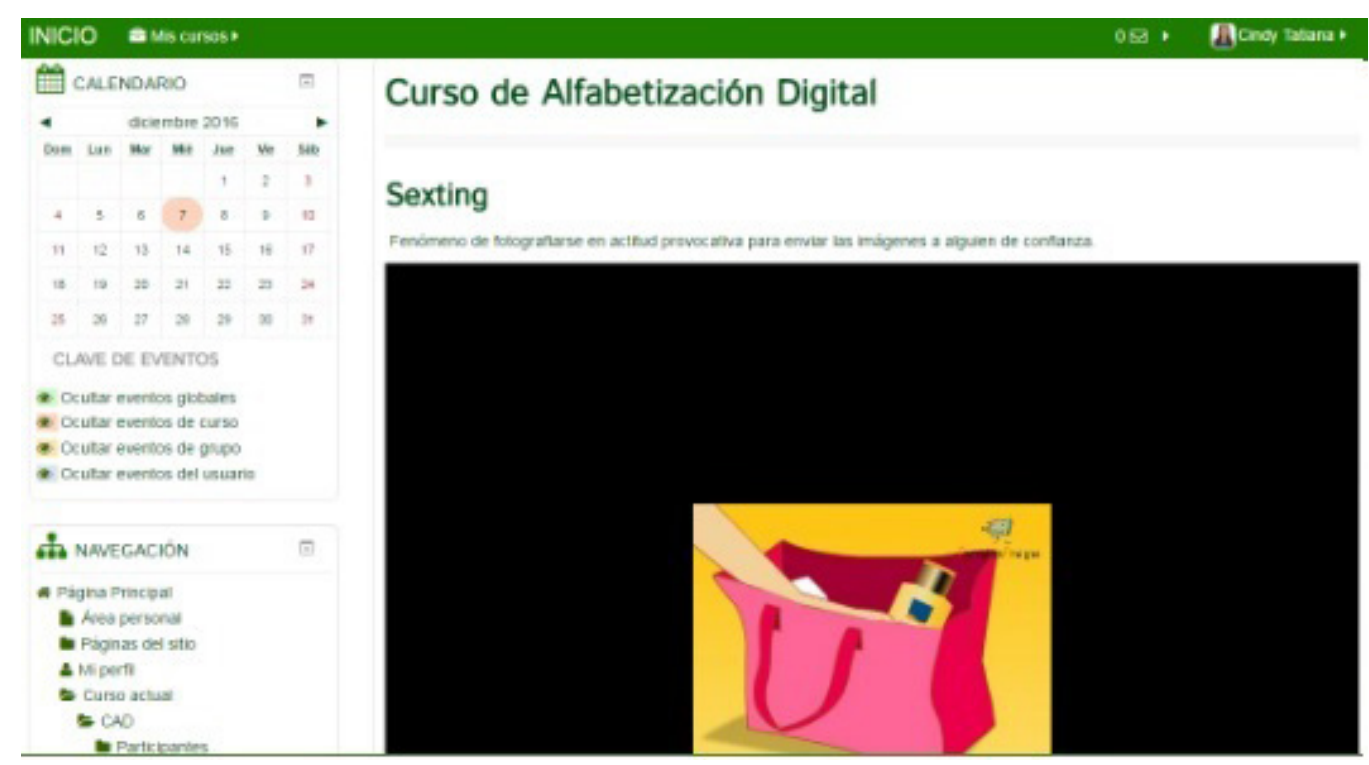

Figura 15. Definición de sexting.

Fuente: (Pantallas Amigas, 2018).

En este mismo aparte se le presenta al estudiante de forma interactiva los conceptos y elementos asociados a los riesgos en el manejo y uso de internet. Terminada esta presentación se asocia un documento de Word con criterios de evaluación para ser desarrollado por los estudiantes e incluirlos en el módulo de evaluación.

En el módulo 3 se encuentran tres actividades relacionadas con el tema de herramientas web 2.0. A continuación, se presenta la descripción de cada una de ellas. 


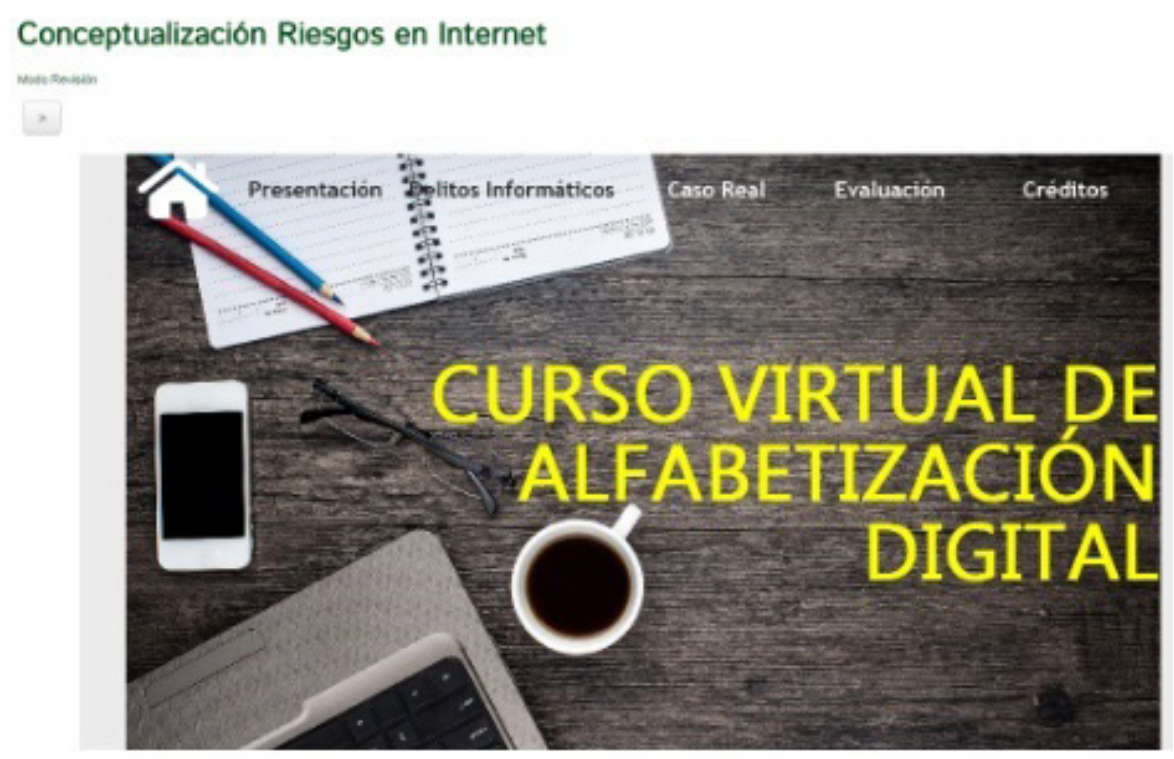

Figura 16. Conceptualización de los riesgos en Internet.

Fuente: elaboración propia.

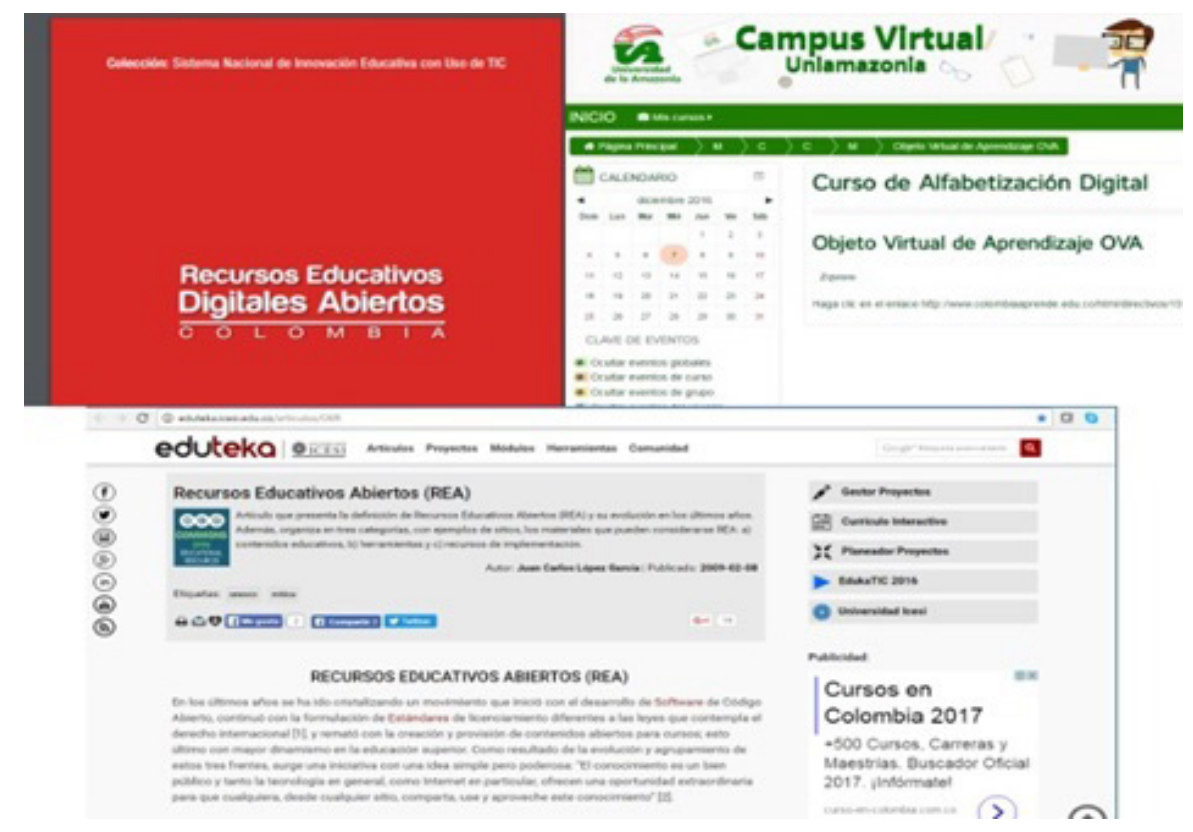

Figura 17. Lectura de recursos educativos digitales abiertos (Reda), objeto virtual de aprendizaje sobre REA y portal de Eduteka.

Fuente: elaboración.

Existen otros elementos para complementar las actividades de formación. En el presente curso virtual se hizo uso de la gamificación y se incluyeron juegos para el aprendizaje y evaluación de las temáticas. En el módulo de actividades académicas se presentan dos juegos de sopas de letras y de acomodar columnas para tratar los temas de ciberacoso y riesgos en internet. La interfaz se puede ver en la figura 18. 


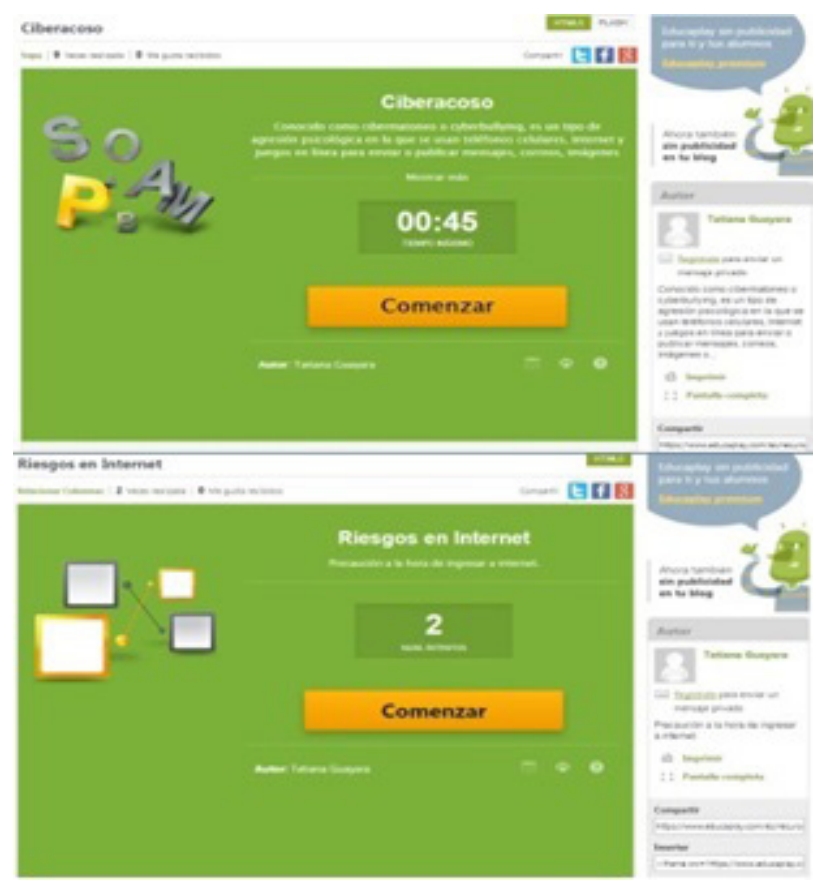

Figura 18. Actividad de sopa de letras ciberacoso y relación de parejas para riesgos en internet.

Fuente: elaboración propia.

\section{Evaluación del curso virtual}

Para la evaluación del curso se presentan dos casos típicos de delitos informáticos y riesgos en internet en los que se reúne todo el contenido del curso. El estudiante se ve enfrentado a estos estudios de caso y se realiza una evaluación cuantitativa y cualitativa de los elementos relacionados con la temática; esto deja entre ver el nivel de apropiación del estudiante de los temas trabajados durante el curso. Adicionalmente, los estudiantes pueden subir material complementario de las otras actividades en los diferentes módulos en el espacio de evaluación y pueden contribuir con material de apoyo para el fortalecimiento del curso.

\section{Conclusiones}

Se logró el diseño y montaje del curso de alfabetización digital para docentes universitarios, logrando ser implementado en la Universidad de la
Amazonia en un conjunto de 100 docentes de un universo de 712 profesionales dedicados a la docencia en el claustro universitario.

La ejecución del curso logró el mejoramiento de las competencias TIC en el grupo docente participantes en el estudio, abarcando temas no comunes como delitos informáticos, hackeo, suplantación de identidad y phishning. El conocimiento de estos temas aporto a la cultura digital en el aula de clase desde el fortalecimiento de las competencias de los docentes.

Los usuarios del curso realizaron una valoración buena del desarrollo del curso y del cumplimiento de sus objetivos. Las orientaciones para el uso del curso virtual favorecieron el trabajo colaborativo y la adquisición de competencias en el uso de herramientas web 2.0.

La incursión de temas como OVAs y REDAs favorecieron y promovieron la curiosidad de los docentes en el uso de estas herramientas para fomentar el trabajo colaborativo entre los estudiantes 
en los diferentes cursos académicos orientados por ellos, el grupo presento un interés particular en los OVAs y preguntaban la necesidad de profundizar en la creación de objetos específicos de sus disciplinas de enseñanza.

El diseño del curso virtual presento diferentes retos a partir de las visiones presentadas por diferentes generaciones de docentes, los cuales tenían diferentes expectativas en el posible resultado y su apropiación en la formación de competencias TIC, en algunos casos los docentes presentaron resistencia al uso de la plataforma virtual pero una vez iniciado la capacitación demostraron un interés sobresaliente por el uso de esta tecnología.

El proyecto deja abierta la posibilidad de la inclusión de otros módulos en el curso para abordar temas de actualidad y formalismo en el uso de las tecnologías de la información y la comunicación para la docencia, además permite tener grados de flexibilidad en los contenidos actuales con el propósito de ser actualizados con nuevo material producido por los mismo docentes o desarrollado en otros ambientes.

\section{Agradecimientos}

A la Universidad de la Amazonia, centro de educación superior ubicado en el Departamento del Caquetá al sur de Colombia. www.udla.edu.co

\section{Referencias}

Binda, N. U. y Balbastre-Benavent, F. (2013). Investigación cuantitativa e investigación cualitativa: buscando las ventajas de las diferentes metodologías de investigación. Revista de Ciencias Económicas, 31(2), 179-187.

Calderón, G., Buitrago, B., Acevedo, M. y Tobón, M. (2013). Competencias TIC para el desarrollo profesional docente. Bogotá: Ministerio de Educación Nacional.

Coll, C. (2014). La evaluación continuada como instrumento para el ajuste de la ayuda pedagógica y la enseñanza de competencias de autorregulación. Cuaderno de Pedagogía Universitaria, 8(15), 14-20.

Espacio, I. (2016). Gestión institucional para la alfabetización digital del docente universitario. Compartir, 5(10).

García, F. C. (2016). Diseño de un modelo curricular E-learning, utilizando una metodología activa participativa. Revista Iberoamericana para la Investigación y el Desarrollo Educativo, 7(13), 147-182. DOI: https://doi.org/10.23913/ ride.v7i13.240

Gilster, P. y Glister, P. (1997). Digital literacy. Nueva York: Wiley Computer Pub.

Iglesias-Rodríguez, A., Olmos-Migueláñez, S., Torrecilla-Sánchez, E. M. y Mena-Marcos, J. J. (2014). Evaluar para optimizar el uso de la plataforma moodle (studium) en el departamento de didáctica, organización y métodos de investigación. Tendencias Pedagógicas, 3, 155-170.

López, M. S. (2015). Aplicación de la plataforma Moodle como herramienta didáctica para el desarrollo de competencias en estudiantes de ingeniería. Revista Iberoamericana para la Investigación y el Desarrollo Educativo, 12.

Ministerio de Educación Nacional (MEN) (2017). Orientaciones_ELearning. Recuperado de https://www.unisabana.edu.co/fileadmin/Documentos/CTA/Orientaciones ELearning.pdf

Olivé, L. (2005). La cultura científica y tecnológica en el tránsito a la sociedad del conocimiento. Revista de la Educación Superior, 4(136), 49-63.

Organización de las Naciones Unidas para la Educación, la Ciencia y la Cultura (Unesco) (2005). Tecnología de la información; tecnología educacional; enseñanza asistida por ordenador; aprendizaje en línea; tendencia educacional; innovación educacional; reforma de la educación; manual del profesor. París: Unesco document.

Rodríguez, A. I., Migueláñez, S. O., Sánchez, E. T. y Marco, J. M. (2015). Evaluar para optimizar 
el uso de la plataforma moodle (studium) en el departamento de didáctica, organización y métodos de investigación. Tendencias Pedagógicas, 23, 155-170.

Salas, M. M., Barrera, S. R. y Fuentes, M. G. (2015). La plataforma Moodle como herramienta de evaluación docente. Revista Iberoamericana para la Investigación y el Desarrollo Educativo, 9.

Tilve, M. D. (2007). ¿Contribuyen las TIC a hacer de los profesores mejores profesionales?: ¿qué dicen los directivos escolares gallegos? PíxelBit. Revista de Medios y Educación, 5-15.

Uniamazonia (2017). Proyecto educativo institucional. Recuperado de http://apps.udla.edu. co/documentos/docs/Consejo\%20Superior/ Acuerdos/2001/Acuerdo\%20031.pdf

Vergel-Ortega, M., Martínez-Lozano, J. J. y Zafra-Tristancho, S. L. (2016). Factores asociados al rendimiento académico en adultos. Revista Científica, 25(2), 206-215. DOI: https://doi. org/10.14483//udistrital.jour.RC.2016.25.a4 